\title{
«Hallo: HIV gibt es noch!»
}

\section{Daniel Lüthi}

Freier Journalist und Fotograf, Medientrainer, Bern

Der 1. Dezember ist der Geburtstag der Aids Hilfe Bern. "Jahre später hat ihn die Weltgesundheitsorganisation WHO zum Welt-Aids-Tag erklärt, es ist wie eine Hommage an uns», sagt Béatrice Aebersold mit einem Schmunzeln. Dieses Jahr feiert ihre Organisation das 30-Jahr-Jubiläum. Mit Freude und Stolz - und in der Überzeugung, dass ihre Beratungen, Schulungen und Aktionen nach wie vor nötig sind.

\section{Irrationales und Rationales}

Aebersold ist fast seit Beginn dabei. Sie hat also den ganzen Wandel in diesem Gebiet und die einzelnen Phasen hautnah miterlebt. So auch die Ausgrenzung in der Anfangszeit. Symbolisch dafür war beispielsweise die Bürosuche Mitte Neunzigerjahre. «Niemand wollte uns», erinnert sie sich, «ein Immobilienbesitzer sagte sogar, ein Büro der Aids Hilfe wäre geschäftsschädigend. Aber eben: Vieles in diesem Gebiet war irrational - und ist es nach wie vor.» Irrational war auch die Angst in der Bevölkerung vor einer Ansteckung. Die ersten Opfer wurden noch in Bleisärgen zu Grabe getragen, «in den Spitälern berührte das Personal Aidskranke nur mit Plastikhandschuhen", erinnert sich Béatrice Aebersold. Es ist eine ganz persönliche und deshalb besonders tiefsitzende Erinnerung.

Sie arbeitete damals, Anfang achtziger Jahre, in einer Institution für Drogenabhängige. Einer ihrer Arbeits-




kollegen war ihr Partner und späterer Ehemann. Einige der Klientinnen und Klienten litten an unerklärlichen Symptomen, an einer unverständlichen Krankheit. 1985, sobald HIV-Tests möglich waren, führte man sie auch in der therapeutischen Gemeinschaft durch, «aus Solidarität machten auch wir Angestellte mit. Was der Befund 'positiv' bedeuten würde, konnten wir allerdings nicht richtig einordnen. Wir Fachleute gingen damals ziemlich unbedarft ans Thema heran", sagt Aebersold. Ganz plötzlich jedoch wurde Abstraktes sehr konkret und Irrationales rational: 12 Klienten und fünf Mitarbeitende liessen sich testen. «Vier Klienten und einer vom Team waren positiv, und ich musste es ihnen mitteilen.» Das Teammitglied war ihr Partner. Von den fünf lebt keiner mehr.

\section{Unwissenheit und Unsicherheit}

"Ja, das war ein Einschnitt», kommentiert Béatrice Aebersold, «die persönliche Betroffenheit wurde prägend für mein Leben, auch wenn für mich der professionelle Bezug zum Thema immer im Vordergrund stand.» Dank ihrer Arbeit hatte sie Zugang zu den Informationen, die damals verfügbar waren, «und ich konnte auf Verständnis und Unterstützung zählen, was damals alles andere als selbstverständlich war.»

\section{«Niemand wollte uns.»}

Weil eben vor allem Unwissenheit und Unsicherheit den Umgang mit der neuen Krankheit prägten. Und Angst. «Es gab Erfahrungen mit anderen ansteckenden Krankheiten, zum Beispiel Tuberkulose, und es gab ein Epidemiengesetz. Über HIV und Aids aber wusste man anfänglich wenig bis nichts. Und die Bedrohung wurde von vielen als grösser dargestellt, als sie für unser Land wirklich war.»

Ziemlich hilflos seien damals, vor dreissig Jahren, auch Ärztinnen und Ärzte gewesen, sagt Aebersold, «auch in der Medizin dominierten Ratlosigkeit und eine grosse Not. Ich erinnere mich genau, wie man mir im Spital sagte, man könne für meinen Partner nichts mehr machen, dies sei ein Akutspital.» Es blieb noch die palliative Betreuung, und dafür gab es zum Glück Institutionen wie das Lighthouse in Zürich. Der Todestag ihres Partners hat sich erst gerade zum zwanzigsten Mal gejährt. Wenn wir uns die Bilder aus der damaligen Zeit vor Augen führen, ist es, als wäre dies alles viel länger her.

\section{Diskriminierung}

Als "Schwulenseuche» wurde Aids in den achtziger Jahren bezeichnet, betroffen von der Infektionskrank-

\section{Béatrice Aebersold}

Béatrice Aebersold wurde 1955 in Konolfingen geboren, wo sie auch die Schulen besuchte. 1974 bis 77 liess sie sich in Interlaken zur diplomierten Pflegefachfrau HF ausbilden. 1980 bis 82 absolvierte sie an der Lindenhofschule Bern das Nachdiplomstudium Pflege, Gesundheitsförderung und Prä-



vention, parallel dazu arbeitete sie in der Gemeindekrankenpflege. 1983 bis 1992 war sie - mit kurzem Unterbruch - in der therapeutischen Wohngemeinschaft für Drogenkonsumierende "Hof Chratten» in Oberbeinwil (SO) tätig. 1988 wurde sie Mitglied der Aids Hilfe Bern, die am 1. Dezember 1985 gegründet worden war. Seit 1996 ist sie deren Geschäftsleiterin. Béatrice Aebersold lebt in Bern.

heit waren in der Tat vor allem homosexuelle Männer. "Schwule reagierten gerade in der Schweiz aber auch schnell und vorbildhaft», erzählt Aebersold, "der erste Präsident der Aids-Hilfe Schweiz war der Fernsehmann André Ratti, er war auch für hetero-normierte Schweizer ein Begriff.» Auch Ratti starb an Aids, wurde zu einem prominenten Opfer der Krankheit. «Er war einer von denen, die einen Lernprozess initiierten und uns ermöglichten, zu informieren und Präventionsarbeit zu leisten.» Vor allem ging es darum, die Leute zu befähigen, sich selber und ihre Partnerinnen und Partner zu schützen. Sehr bald entstanden in einzelnen Kantonen, so auch in Bern, regionale Beratungszentren. "Die Aids Hilfe hatte Antworten auf drängende Fragen, dies trug zur Beruhigung bei. Wir konnten Missverständnisse klären und Diskriminierung vermindern.» Wir: Das waren damals vor allem Männer. «Als die Aids Hilfe Bern Mitte neunziger Jahre zu einer professionellen Organisation wurde und man in Bern eine Geschäftsleitung etablieren wollte, bewarb ich mich für die Leitungsstelle. Viele schwule Männer goutierten das nicht, ich musste mich als Frau durchsetzen und doppelt beweisen - es war sehr schwierig.»

\section{Die grosse Wende ...}

Der entscheidende Umbruch kam 1996: Die Krankheit, die bisher als tödlich galt und es für Tausende auch war, wurde therapierbar. «Da gab es biblische Szenen - Leute, die dem Tod im letzten Moment ab der Schippe sprangen", erzählt Béatrice Aebersold. Die neue Therapie bedeutete neue Hoffnung und Sicherheit. «Inzwischen wissen wir, dass eine Therapie auch eine präventive Wirkung hat - ein grosser Erfolg." Aber - da ist ein Aber. «Mit dem Greifen der Therapie stieg die Zahl der Neuinfektionen wieder an. Das hatte auch mit zunehmender Unachtsamkeit zu tun. Der Beginn der The- 
rapierbarkeit einer HIV-Infektion bedeutete unter anderem das Ende einer Bedrohung. Nach Jahren der Disziplin und Abstinenz schalteten viele ihr Hirn in bestimmten Situationen wieder aus.»

Heute habe sich alles normalisiert, sagt Aebersold, «die Situation ist absolut nicht mehr vergleichbar mit damals. 70 Prozent der HIV-infizierten Menschen arbeiten, mehrheitlich in einem Vollpensum und mit ihrer

«Da gab es biblische Szenen -

Leute, die dem Tod im letzten Moment ab der Schippe sprangen.»

Infektion unerkannt.» Und wieder ein Aber: «Die Diskriminierung von Menschen mit HIV ist nach wie vor gross, am Arbeitsplatz, bei Versicherungsfragen, aber leider auch immer wieder im privaten Umfeld.»

\section{... und die grosse Frage}

Trotzdem: Vieles hat sich in diesem Bereich - markant teilweise - zum Positiven entwickelt. Die Grundsatzfrage sei deshalb erlaubt: Braucht es die Aids Hilfe noch? «Ja», sagt Béatrice Aebersold, «uns braucht es nach wie vor.» Und fügt hinzu: "Mittelfristig zumindest.» Verändert habe sich der Einsatzbereich ihrer Organisation: «Zu den speziell vulnerablen Gruppen gehören heute vermehrt Migrantinnen und Migranten. Menschen aus Subsahara-Afrika, aber auch aus den ehemaligen GUSStaaten. In Russland zum Beispiel nehmen die Infektionen beim Spritzentausch dramatisch zu.» Die Aids Hilfe Bern zählt laut ihrer Geschäftsleiterin 200 direkt betroffene Klientinnen und Klienten pro Jahr, die Hälfte davon stammen aus dem Ausland. «Wir haben Projekte im Bereich MSW (Male Sex Work) und sprechen spezifisch auch Freier an.» Beratungen und Schulungen nehmen unter anderen Angehörige in Anspruch, Lehrpersonen - und Fachleute. Pflegende, oder ab und zu auch Ärztinnen und Ärzte. «Mit Infektiologen arbeiten wir sehr gut und zum Teil eng zusammen», lobt Aeber- sold, "wir unterstützen sie beispielsweise in rechtlichen oder sozialen Fragen.» Weniger positiv beurteilt sie die Grundlagen von Allgemeinmedizinern: «Hausärztinnen und Hausärzte sprechen - die Reproduktion ausgenommen - zu wenig über Sexualität. In ihren Praxen vergessen sie dann auch allzu oft, eine Sexualanamnese zu machen und einen HIV-Test durchzuführen. Die Vielfalt des sexuellen Lebens haben sie zu wenig im Blick. Unter anderem auch deshalb ist die Zahl der sogenannten 'Late Presenters', der zu spät diagnostizierten HIV-Infektionen, mit rund 50 Prozent viel zu hoch.»

Mit der Abnahme der HIV-Aids-Problematik habe sich dieser negative Aspekt verstärkt. «Oft müssen wir Ärztinnen und Ärzten sagen: Hallo, HIV gibt es noch! Möglicherweise auch bei euren Patientinnen und Patienten.» Aebersold verweist auf die aktuellen Zahlen: 2014 wurden in der Schweiz rund 520 Neudiagnosen gestellt, 60 davon allein im Kanton Bern. Weltweit und auf das gleiche Jahr bezogen gehen die Behörden von rund 2 Millionen Neuinfektionen und rund 1,2 Millionen Todesfällen aus.

Persönlich ist Béatrice Aebersold des Themas nicht überdrüssig geworden. «Natürlich gibt es nach all den Jahren etliche Déjà-Vus», sagt sie, «immer wieder aber habe ich auch neue spannende Erlebnisse.» So habe sie letzthin das grosse Vertrauen einer Klientin aus Westafrika an ihre eigenen beruflichen Wurzeln erinnert: «Ich hatte damals den Berufswunsch Krankenschwester, weil ich in Afrika arbeiten wollte, im Spital von Albert Schweitzer zum Beispiel. Jetzt ist Afrika zu mir gekommen.»

\section{Die nächste Begegnung mit ...}

Am Ende jeden Monats stellt die Schweizerische Ärztezeitung eine Persönlichkeit vor, die sich im Gesundheitswesen engagiert. Im Dezember schildert Daniel Lüthi seine Begegnung mit Cyrille Bocion, stellvertretender Oberarzt im Ambulatorium des städtischen Dienstes Zürich. 